Diestro Fernández, A, Ruiz Corbella, M. y Galán, A. (2017). Calidad editorial y científica en las revistas de educación. Tendencias y oportunidades en el contexto 2.0. Revista de Investigación Educativa, 35(1), 235-250 DOI: http://dx.doi.org/10.6018/rie.35.1.244761

\title{
Calidad editorial y científica en las revistas de educación. Tendencias y oportunidades en el contexto $\mathbf{2 . 0}$
}

\section{Scientific and editorial quality in educational journals. Trends and opportunities in the 2.0 context}

\author{
Alonso Diestro Fernández*, Marta Ruiz-Corbella*y Arturo Galán** \\ *Departamento de Teoría de la Educación y Pedagogía Social. UNED \\ ** Departamento de Métodos de Investigación y Diagnóstico de la Educación. UNED
}

\begin{abstract}
Resumen
Las revistas científicas han sufrido una fuerte revolución en la última década, impulsadas por el logro del mayor nivel posible de visibilidad e impacto internacional. Se trata de un proceso mediatizado por las oportunidades y los cambios que se derivan del nuevo contexto de la Science 2.0 y el Open Access. Este trabajo analiza la necesidad de recurrir a una valoración más global y amplia del impacto que la proporcionada por los rankings publicados por las grandes editoriales basadas exclusivamente en las citas (JCR y SJR). El choque de tendencias se hace evidente en este contexto de herramientas 2.0, en el que las posibilidades de rastreo y medición bibliométrica, plagada de nuevas aplicaciones y herramientas, se multiplican exponencialmente en el entorno digital. El artículo evidencia las grandes diferencias de oportunidades entre las revistas científicas de educación en el contexto iberoamericano y el anglosajón, desigualdad que podría reducirse si se introducen otras métricas para medir el factor de impacto de una publicación.

Palabras clave: revistas científicas; investigación en educación; gestión editorial; métricas; calidad editorial.
\end{abstract}

Correspondencia: Alfonso Diestro Fernández, adiestro@edu.uned.es, UNED, Facultad de Educación, Departamento de Teoría de la Educación y Pedagogía Social, C/ Juan del Rosal, 14, 28040, Madrid. 


\begin{abstract}
Scientific journals have undergone a significant revolution in the last decade in the pursuit of achieving the highest possible level of international exposure and influence. This process is clearly determined by the changes and opportunities derived from the new context offered by Science 2.0 and Open Access. This paper analyses the need for a more comprehensive and broad assessment of impact that are provided by the published rankings by the big publishers based exclusively on citations (JCR and SJR). An evident clash of trends takes place within such context of 2.0 Tools in which the possibilities of bibliometric search and analysis, with its countless means and applications, multiply exponentially in the digital environment. This article shows the great differences of opportunities between the scientific education journals of Iberian and Anglo-Saxon contexts, an inequality that could be reduced if other metrics are introduced to measure the impact factor of a publication.

Keywords: scientific journals; education research; editorial management; metrics; quality assurance.
\end{abstract}

\title{
Introducción
}

Las revistas científicas, como medio de difusión de los avances de la investigación, son uno de los ámbitos de la comunicación que más han evolucionado en la última década, especialmente en los aspectos formales, técnicos, económicos y evaluativos. El foco de preocupación está pasando de la calidad científico-formal a la consecución del mayor nivel posible de visibilidad e impacto internacional. Por ello, el objetivo principal de este trabajo responde a tres aspectos: en primer lugar, describir y analizar las nuevas formas de difusión de las revistas científicas de educación ante las oportunidades y los cambios que se derivan del nuevo contexto de la Science 2.0 (Open Science) y el Open Access (OA); acto seguido, valorar la pertinencia de la utilización del Factor de Impacto (FI) para la consideración de las revistas iberoamericanas de ciencias sociales, frente a las medidas emergentes basadas en otros indicadores. Y, por último, aportar evidencias sobre la desigualdad de oportunidades entre el contexto anglosajón y el iberoamericano a la hora de situar revistas en las bases de datos selectivas más relevantes.

En este estudio se analizarán los aspectos diferenciadores que determinan el valor editorial y científico de las revistas de educación. Algunos de ellos son controvertidos porque califican a las publicaciones científicas de diferente forma, tanto cuantitativa como cualitativamente, principalmente a partir del FI $-n^{\circ}$ de citas / $n^{\circ}$ de artículos publicados-. En las últimas décadas han proliferado todo tipo de indicadores bibliométricos y cienciométricos, destacando el Índice $\mathrm{h}(\mathrm{Ih})-\mathrm{n}^{\mathrm{o}} \mathrm{N}$ de artículos con $\mathrm{n}$ citas- como uno de los más recientes, que compite directamente en importancia con el FI, aunque midan aspectos bibliométricos diferentes. Esto nos brinda la oportunidad de valorar si la importancia otorgada al FI o al Ih a nivel global -propia de las ciencias puras y biosanitarias- resulta equitativa con las revistas de educación, al provenir de áreas de conocimiento con tradiciones editoriales, científicas y contextuales completamente diferentes. De hecho, la investigación pedagógica abarca estudios empíricos, hermenéuticos, históricos, revisiones bibliográficas, etc., que exigen tratamientos metodológicos diferenciados. En consonancia con lo anterior, y a diferencia de otras ciencias, en el 
campo de la educación (y sobre todo en las áreas no empíricas) se cuenta con una financiación económica mucho menor, por lo que resulta aún más complicado plantear grandes proyectos y conseguir financiación externa en convocatorias públicas competitivas. Pese a todo, se obtienen resultados novedosos que hacen avanzar la ciencia.

En este contexto particular, en el que tratamos con un campo científico relativamente nuevo y pequeño, sobre todo en comparación con las ciencias experimentales, es importante analizar si existen formas alternativas (o complementarias) más adecuadas para valorar el impacto de las ciencias sociales y, en particular, de la pedagogía, como, por ejemplo, los altmetrics (Authors Level Metrics), que permitan evaluar otro tipo de variables más allá del número de citas y que podrían ser más adecuadas (y equitativas) a este campo científico. Dichas variables reflejan un impacto de otra naturaleza, es decir, un impacto social basado también en valores de difusión (lecturas, descargas, accesos, interacciones en RR. SS, etc.) pero que puede ser tan importante para las ciencias sociales como el FI para las ciencias experimentales.

En definitiva, nuestro análisis se centra en demostrar la necesidad de recurrir en las ciencias sociales a una valoración más global y amplia del impacto que el proporcionado por los rankings publicados por las grandes empresas editoriales (el JCR de Thomson Reuters y el SJR de Elsevier), basados principalmente en las citas contabilizadas en las revistas indizadas en sus propios productos y catálogos. Además, la utilización exclusiva de tal medición del impacto se hace cuestionable en el contexto de la Open Science, el Social Media y las herramientas 2.0, por sus mayores posibilidades de seguimiento y medición bibliométrica en el contexto digital. Aparecen, a la vez, nuevos modelos de comunicación y difusión basados en redes sociales (RRSS) como Facebook, Twitter, blogs, etc., y portales específicos dirigidos a investigadores como ResearchGate, Academia.Edu, Mendeley o Google Scholar. Incluso algunos de ellos han desarrollado nuevas métricas, variables e índices relativos de difusión, impacto y seguimiento, tanto de las revistas como de los propios autores-investigadores (Ruíz-Corbella, Galán \& Diestro, 2015); aunque aún deben ganar en fiabilidad y precisión, abren un nuevo abanico de posibilidades.

Se vislumbra, pues, un nuevo horizonte editorial y científico en el que, si se quiere competir con los mejores, se exigirá dominar estas herramientas a la par que se avanza en instrumentos para medir la calidad y el impacto de las publicaciones con base en nuevos indicadores más complejos que el FI.

\section{Open Access y Science 2.0: implicaciones editoriales}

El cambio de paradigma científico hacia la denominada Ciencia 2.0 es fruto de una realidad que se apoya en los rápidos y dinámicos cambios provocados por la consolidación de la revolución digital, la innovación tecnológica, el OA y la adopción de una cultura 2.0. El nuevo modelo de ciencia persigue una mayor y más amplia transferencia del conocimiento desde una perspectiva aperturista y globalizada. Reconoce la importancia de la responsabilidad y la rendición de cuentas, basadas en la transparencia y el acceso a los datos, y de la innovación que supone la nueva manera de hacer, comunicar, difundir y hacer sostenible su coste: “La Ciencia 2.0 no sólo 
tiene grandes implicaciones en la manera de hacer ciencia, sino que también podría tener un importante impacto social y económico" (European Commission, 2014, p. 6).

Nunca hasta ahora se dispusieron de semejantes cotas de inmediatez, accesibilidad, elaboración, interacción, re-producción y emisión de información y de contenidos, en cualquier momento y lugar. Además, en la mayor parte de los casos, los servicios del contexto 2.0 son gratuitos, masivos, funcionales y altamente accesibles para los usuarios. Recordemos que Europa, en su reciente estrategia Science 2.0, identifica la tecnología digital, el cambio de las prácticas de investigación y su impacto, como las claves de este nuevo modo de "hacer ciencia" (European Commission, 2015).

A la vez, iniciativas internacionales, como DOAJ (Directory of Open Access Journals) o Sherpa Romeo, velan por la promoción del OA y establecen diferentes niveles de apertura de estas publicaciones, aumentando el impacto social y científico de los resultados de la investigación mediante una mayor visibilidad. Todo este movimiento ha generado varias declaraciones y convenios internacionales, que marcan las tendencias hacia el aperturismo editorial y nuevas métricas de la evaluación científica como, por ejemplo, la PLoS: Open letter to publishers (2001), las Declaraciones de Budapest (2002), Bethesda (2003) y Berlín (2003), la reciente iniciativa de la Declaración de San Francisco sobre Evaluación de la Investigación (DORA, 2012), la propia estrategia del Open Science de la UE (2014) o la Declaración de Leiden sobre métricas de la investigación (2014).

El punto neurálgico en todo este proceso es la especial atención que se ha de prestar a la convergencia entre el enorme potencial del OA, las nuevas implicaciones del Science 2.0 y las políticas que se derivan de ello. Por ejemplo, la Agencia Digital para Europa ha establecido una ambiciosa agenda relacionada con el OA y la financiación de proyectos. Así, la UE, en su Programa Marco de 2014, obliga a que todas las publicaciones financiadas con sus fondos, generalmente artículos, se publiquen en revistas OA mediante la ruta dorada (acceso inmediato para todos). Incluso sugieren a los Estados miembros que adopten un planteamiento similar, con el objetivo de que el $60 \%$ de los artículos de la investigación financiada con fondos públicos europeos pueda consultarse libremente para 2016. España recoge esta indicación en la última convocatoria del Plan Nacional de $I+D+I$ (2015), que se deriva de la Ley 14/2011 de la Ciencia, la Tecnología y la Innovación. No obstante, sin renunciar a todas las posibilidades que proporciona el OA, la enorme visibilidad que facilitan sus tecnologías no es suficiente. Las acciones coordinadas de la política de la ciencia en nuestras regiones será crucial para respaldarlas e impulsarlas (Vessuri, Guédon \& Cetto, 2013). Y esto sin entrar en las "imposturas en el ecosistema de la publicación científica", que está generando un "mercado de artículos", pervirtiendo el propio sentido de estas publicaciones y olvidando "ahondar en los detalles que marcan la calidad de todo el proceso de selección y publicación de artículos, independientemente del modelo de negocio de la revista y de las fuentes que la recojan" (Giménez-Toledo, 2014, p. 14).

Ahora bien, Sánchez Perona (2015) destaca la peligrosa deriva que este cambio puede provocar ¿Cómo es posible conjugar la presión y la necesidad por publicar en revistas de alto impacto, mantener el sistema de suscripciones de las revistas y el imperativo de la publicación de resultados financiados con fondos públicos en OA? Las políticas públicas pueden provocar que el sistema de financiación por suscripciones de algunas revistas pierda sentido por la obligatoriedad del OA. En consecuencia, empiezan 
a aparecer nuevas y diversas fórmulas de gestión económica (nuevos modelos de negocio), que garanticen la sostenibilidad, la adaptabilidad y la viabilidad económica de estas revistas (Villarroya, Claudio, Abadal \& Melero, 2012), entre las que se están imponiendo tres tendencias:

- Todos los autores deben pagar por publicar. Normalmente se trata de una cantidad no demasiado elevada, en torno a $100 €-300 €$, pero aún así es un coste que puede provocar falta de equidad a nivel global.

- Los autores pueden realizar un pago opcional para que la publicación esté en abierto desde el primer momento, incluso en la modalidad published online in advance. Este pago contribuiría a cubrir los ingresos que la revista dejaría de percibir por la venta de artículos a particulares o a bases de datos. En este caso, las cantidades que solicitan las revistas son muy variadas, entre $200 €$ y $3.000 €$, dependiendo del país y la empresa editorial. Este pago voluntario es más coherente con las políticas europeas de investigación y publicación en OA.

- La venta de números monográficos a grupos de investigación o a determinadas entidades. Esta práctica es más controvertida, especialmente si el control de las revisiones de los artículos se cede al "grupo comprador".

El coste por publicar, como consecuencia del nuevo modelo de negocio de las revistas científicas y del OA, está causando un gran interés en la literatura reciente, ya que es difícil estimar el precio medio por artículo en cada área de conocimiento y en cada región editorial. El Informe Finch (2012, p. 61) estima el coste de publicación de un artículo científico en el Reino Unido, incluidos los cargos del proceso editorial (las tasas article processing charges -APC-), entre 1500£-2000£. Nassi-Caló (2013) señala que los costes de APC en conocidas revistas internacionales en OA oscila entre 100\$-3000\$, y en algunas revistas híbridas entre 3000-5000\$. En esta perspectiva, López-Torres (2015) advierte que los precios llegan a fluctuar entre $500 €$ y $2.500 €$ por artículo, "correspondiendo los más elevados a revistas de editoriales internacionales con alto factor de impacto y siendo aún más elevados cuando se trata de revistas híbridas" (p. 180).

Una reciente investigación de Mañana-Rodríguez y Giménez-Toledo (2015) valoran la relación entre el coste del artículo publicado y el valor añadido, en términos de desempeño bibliométrico, de las revistas editadas por entidades con ánimo de lucro y con un alto coste por artículo, el precio por cita, el índice compuesto de precio y el índice relativo de precio de entre las revistas de educación en JCR, destacando que, la relación entre ellas coste y valor añadido, es claramente cuestionable:

La publicación de artículos en revistas costosas no implica una mayor probabilidad de contar con valor añadido a esa publicación específica, y se subraya el papel de las revistas menos costosas en el proceso de comunicación científica. Asimismo, también ponen en cuestión cuál es el valor añadido de las revistas más costosas y los grupos editoriales que las publican, y por lo tanto también pone en entredicho el equilibro en el mercado de revistas científicas en Educación (p. 53). 
Todo ello nos lleva a confirmar que muchas publicaciones "menores" están en desigualdad de condiciones frente a las revistas mejor situadas o a las grandes empresas editoriales. En nuestro país, en el momento actual, la mayor parte de las revistas no están en disposición de cobrar por publicar, no porque su actividad se ejerza sin ánimo de lucro, sino porque no existe una cultura entre los autores de preocuparse por los costes de la publicación. En consecuencia, una revista "media" que cobre por publicar perdería muchos originales ante una alternativa en la que se pueda publicar de forma gratuita.

A la vez, si nos comparamos con las revistas anglosajonas editadas por la grandes empresas editoriales (Elsevier, Taylor E Francis, etc.), la primacía científica y lingüística y su control del mundo editorial en todas las áreas de conocimiento les permiten disponer de un mayor número de revistas en catálogo (por suscripción o en OA), pudiendo ampliar la estrategia empresarial basada en el aumento de la producción de nuevos títulos en OA y relacionar su marketing con la reputación de algunas de sus revistas más prestigiosas de pago por suscripción.

\section{Criterios de evaluación de revistas y digitalización}

Gracias a este cambio de paradigma hacia la ciencia 2.0, las revistas científicas se han convertido en el canal de comunicación de la investigación más destacado, reconocido y privilegiado, al actuar como instrumento fundamental en la organización, vertebración e institucionalización social de una disciplina (Delgado, Ruiz-Pérez \& JiménezContreras, 2006). Este respaldo proviene, como destacan Galán (2014) y Giménez-Toledo y Tejada-Artigas (2014), de los poderes públicos y las políticas de evaluación de la productividad investigadora instauradas en los últimos 25 años, tanto a nivel nacional como internacional. Dichas políticas han primado la publicación de los resultados en las revistas científicas de impacto (las incluidas en el JCR principalmente), con lo que éstas adquirieron un notable peso en los procesos de acreditación, evaluación y reconocimiento profesional. Sin embargo, la escasez de revistas españolas de ciencias sociales, jurídicas y humanidades en dichos índices dio lugar a nuevas iniciativas para dilucidar los criterios de calidad en estas áreas de conocimiento (Hernández Pina \& Maquilón, 2010). Aunque no ponemos en duda su buena intención, no siempre parten de una adecuada y completa información ni se aplican correctamente. "Corremos el riesgo de dañar el sistema con las mismas herramientas diseñadas para mejorarlo, si la evaluación se lleva a cabo cada vez más por organizaciones sin un bagaje de buenas prácticas o desconociendo cómo interpretarlas" (Hicks, Wouters, Waltman, De Rijke \& Rafols, 2015, p. 1).

Si analizamos las revistas científicas de educación en los últimos 15 años, observamos una rápida transición de las publicaciones impresas al formato digital, favorecidas por cuestiones de escasez u optimización económica y un incremento de nuevas revistas en formato digital y en OA (nacidas ya atendiendo a los criterios internacionales de calidad editorial). Al mismo tiempo, el número de publicaciones científicas publicadas exclusivamente en formato impreso se ha reducido de forma drástica. Valga como referencia que en 2014 se editaban 168 revistas en formato digital frente a 31 impresas (Ruíz-Corbella, Galán \& Diestro 2014). Esto demuestra que el salto hacia modelos de 
edición digital fue rápidamente percibido como un canal especialmente atractivo para difundir la ciencia. El crecimiento de proyectos editoriales basados en gestores y sistemas digitales como el OJS (Open Journal System) fue exponencial. D'Antonio (2014) afirma que, de 2004 a 2014, el número de revistas vivas que utilizan este gestor pasó de 1930 a 7000, al facilitar sustancialmente su gestión, sostenibilidad y difusión. De esta forma, la digitalización de las revistas científicas de educación ha generado numerosos beneficios: una mayor área de influencia, inmediatez, almacenamiento, reducción de costes, etc., lo que ha supuesto, en la última década, más que una tendencia, un cambio de paradigma (Aliaga, 2014).

Torres-Salinas (2008) mantiene que, en lo relativo a esta visión 2.0, "se enmarcan todas aquellas utilidades presentes en el portal de la revista o la participación de la misma en comunidades y redes sociales" (p. 25). Entre dichas utilidades diferencia los elementos 2.0 internos, relacionados con la edición (interoperabilidad, múltiples formatos), de los externos, que se corresponden con la difusión y visibilidad (blogs, RRSS, foros, chats, comunidades, etc.). Aunque las revistas en educación se han digitalizado de manera considerable en la última década, todavía son pocas las que cuentan con estos elementos y con una visión global de los procesos editoriales y científicos de calidad en la perspectiva del 2.0. Si los equipos editoriales no se modernizan en este sentido, se podría perder una nueva oportunidad de aumentar la competitividad y visibilidad, a partir del momento en el que se comiencen a valorar las nuevas métricas o nuevas variables -una vez ganen la fiabilidad y reputación suficientes- basadas en estos datos.

\section{La brecha entre las revistas iberoamericanas y las anglosajonas}

Ya hemos comentado algunas razones de la gran brecha entre las revistas iberoamericanas y las anglosajonas, como los recursos económicos, el idioma o el acceso a las grandes bases de datos internacionales, aspectos tan básicos que casi cercenan toda posibilidad de igualación. De hecho, gran parte de las revistas presentes en el Social Sciences Citations Index (SSCI) ubicadas en el campo de Education E Educational Research -prácticamente dos tercios del total- pertenecen a las grandes empresas y multinacionales editoriales con intereses en el sector: Elsevier, Springer, Sage, Routledge, Taylor E Francis, Wiley, Chicago Press, etc. Si ubicamos geográficamente a estas revistas, encontramos una fuerte presencia anglosajona (Gran Bretaña, Estados Unidos, Australia y Nueva Zelanda, con 182 de 224) en la figura 1. Además, la gran mayoría de los títulos presentes en el catálogo emplean el inglés como lengua principal (y exclusiva) de publicación, aunque no sea la lengua propia del país, como en el caso de Holanda.

La Figura 2 incide en la escasa presencia de las revistas iberoamericanas en los dos rankings más prestigiosos.

Ruíz-Pérez, Martín-Martín y Delgado López-Cózar (2015) ponen de manifiesto la importancia de los cambios que han debido asumir en los últimos años todas las revistas científicas españolas, para cumplir con la adaptación a los criterios determinados por las agencias nacionales e internacionales de evaluación de la calidad de la ciencia. Mendoza y Paravic (2006) se expresaban en términos parecidos en lo referido a las 


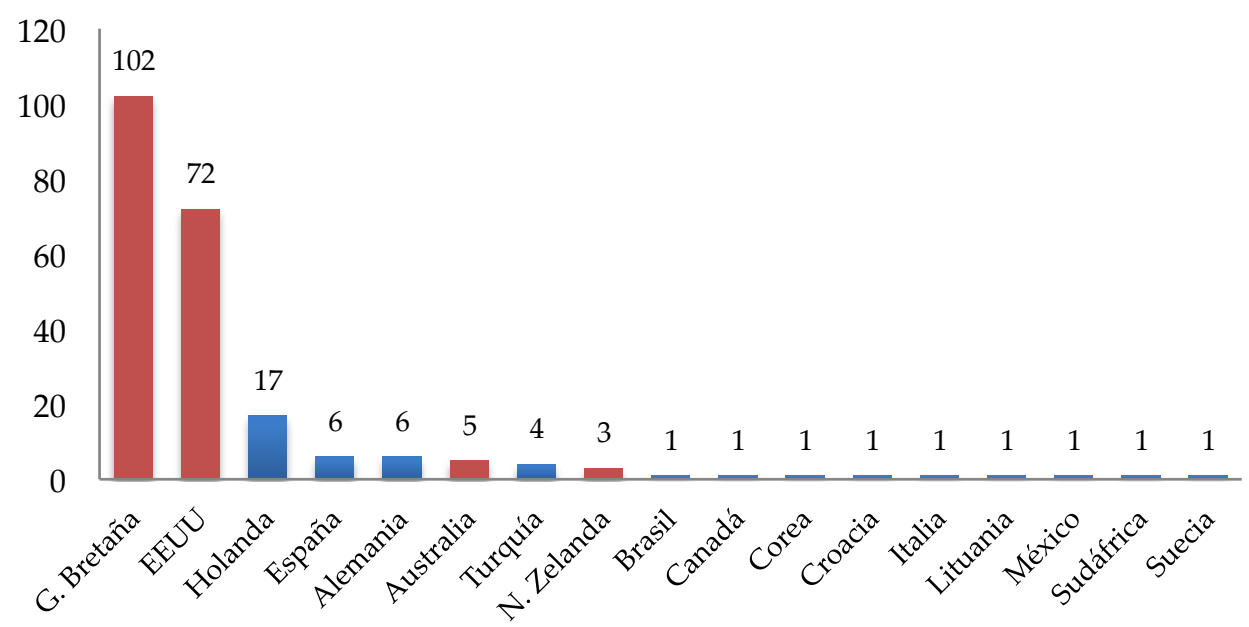

Figura 1. Distribución del número de revistas presentes en el SSCI-JCR por países del área Education \& Educational Research. Elaboración propia a partir del SSCI-JCR (2014)



Figura 2. Presencia de revistas científicas de educación en las bases de datos de SCOPUS y SSCI - JCR, 2015. Elaboración propia a partir de SJR (2015), SSCI-JCR (2015) y WoS (2015)

publicaciones latinoamericanas. Sin embargo, de acuerdo con Abadal y Rius (2006), todavía hay muchos aspectos que deberían ser abordados para potenciar la presencia en WoS y Scopus (los dos grandes referentes en los que la mayoría convergen, Giménez-Toledo, 2015), entre los que destacamos: mejorar la digitalización de contenidos; perseguir la inclusión en portales, repositorios, catálogos, bases de datos y buscadores especializados; profundizar en la difusión de los contenidos mediante el OA; fomentar el empleo de versiones multilingües de los artículos y mejorar la comunicación de las novedades, así como el seguimiento y la medición de las estadísticas de la audiencia. 
Recientemente, las propias universidades, conscientes de la importancia de las revistas, han concedido más apoyo documental e informático para su digitalización, implicando a nuevos agentes de diversas disciplinas (informáticos, documentalistas, bibliotecarios, etc.). Este paso significativo resulta aún escaso ante la ingente tarea que debe abordar cada equipo editorial, especialmente cuando lo comparamos con los recursos humanos y materiales de los grandes grupos editoriales anglosajones.

A pesar de la insistencia de las políticas científicas de primar las publicaciones de WoS y Scopus, no olvidemos que Thomson Reuters y Elsevier son multinacionales privadas que, con sus propios intereses económicos, están ejerciendo como lobbies del sector editorial universitario y científico. Rozemblum, Unzurrunzaga, Banzato y Pucacco (2015) llegan a afirmar que es dudosa la utilización de WoS para la evaluación de las ciencias en general, pero que en el caso de las ciencias sociales es inaplicable.

Editores y autores iberoamericanos deben afrontar que la profesionalización de las revistas supone enfrentarse a las propias particularidades contextuales. Hace ya una década, Delgado et al. (2006) destacaban que las revistas españolas presentes en la escena internacional están ahí por la ilusión y el tesón de un pequeño grupo de investigadores, no por un diseño editorial profesional.

Esta falta de profesionalización es otra de las causas de la brecha entre el contexto anglosajón y el iberoamericano. Prueba de ello es que de las 55 revistas españolas de educación que cumplen con los 33 criterios LATINDEX, 45 de ellas (el 81,8\%) están ligadas a la universidad en cuanto a titularidad editorial (Ruíz-Corbella et al., 2014). En esta misma línea, Rodríguez-Yunta y Giménez-Toledo (2013) analizan las debilidades del sector editorial de humanidades y ciencias sociales, detectando un predominio de las publicaciones de carácter institucional o generadas por un pequeño colectivo (asociación, departamento, grupo de investigación) con una baja producción anual, un escaso impacto y una gestión poco eficaz, que encuentra dificultades para superar cierta imagen de endogamia. Ante estas carencias, y visto el elevado número de revistas españolas de educación (más de un centenar, 133 según MIAR, o 137 en la clasificación de la ANEP, según DICE), mientras que sólo 6 están en SSCI, parece necesario plantear nuevos modelos de gestión y edición de esas publicaciones.

\section{De la calidad editorial a la valoración de la calidad científica y la visibilidad de las revistas}

Lo que subyace en las diversas iniciativas de evaluación de la calidad de las revistas, ya sea a nivel internacional -como es el caso de LATINDEX (1995) y RedALyC (2002)o nacional -IN-RECS, RESH, DICE (extintos), CIRC, MIAR o, más recientemente, el Sello de Calidad de la FECYT (2006)-, es el logro de publicaciones competitivas y de calidad externamente contrastada. A mediados de los noventa se establecieron todo tipo de criterios e indicadores que pretendían valorar y categorizar a las revistas en función de su calidad editorial y científica. En poco tiempo se pasó de los 36 criterios agrupados en 4 categorías que ofrecía LATINDEX para revistas electrónicas, a los 53 criterios agrupados en 5 categorías que establecía FECYT. A diferencia de LATINDEX, FECYT identifica no solo los aspectos formales, sino también otros como la calidad informativa como medio de comunicación científica, la calidad del proceso editorial, 
la capacidad de atracción y calidad científica, la calidad de difusión, audiencia y visibilidad y la calidad de repercusión (Hernández Pina y Maquilón, 2010). En el caso de RedALyC son 60 los criterios empleados, pero, como indican Rozemblum et al. (2015), el desgranamiento excesivo que propone RedALyC obliga a los diferentes editores a ceñirse a una uniformidad que anula las particularidades de cada revista, sin ganar en "calidad científica", que es su objetivo principal.

En mayor o menor medida, existe un acuerdo internacional sobre los aspectos que determinan la calidad editorial de las revistas científicas. Otra cosa bien distinta es el valor o la ponderación que tengan cada uno de los indicadores según la base de datos o el sistema de catalogación de referencia. Un reciente estudio realizado por Chavarro (2013) compara los criterios de calidad editorial, de gestión y de contenido de LATINDEX con los de RedALyC, SciELO, Scopus y WoS, y concluye que, en términos de calidad editorial, todas son muy similares, independientemente del sistema de indexación. No en vano, como destacan Ruíz-Corbella et al. (2014), una gran mayoría de revistas aún no ha logrado todos los estándares de calidad editorial. Por ejemplo, de las 137 revistas científicas españolas de educación reconocidas por la ANEP (Agencia Nacional de Evaluación y Prospectiva), sólo 55 de ellas cumplían en 2012 con los 33 criterios de calidad editorial de LATINDEX para revistas impresas. A mayor abundamiento, aunque añadiendo criterios basados en citas, en 2015, únicamente 19 revistas han obtenido el Sello de Calidad Científica de la FECYT y sólo 6 están presentes en el JCR. No obstante, una buena noticia del trabajo sostenido que se está llevando a cabo en este sector es que, en el nuevo producto de Thomson Reuters (aún en construcción) denominado Emerging Sources Citation Index (ESCI), están ya incorporadas 29 revistas españolas (Galán, 2016).

Los indicadores formales de calidad editorial son comunes a los distintos índices y relativamente sencillos de conseguir pero, ¿implican realmente calidad científica? ¿O deben prevalecer las citas como el indicador diferenciador de calidad? Rozemblum et al. $(2015$, p. 76$)$ definen la calidad científica como

aquella que a través de la evaluación por pares y la exigencia de originalidad de los trabajos publicados es controlada por el colegio invisible; es decir, por grupos de especialistas en una disciplina que evalúan la producción científica y que, además, aportan contribuciones para el crecimiento y sostenimiento de las revistas, que difunden los resultados de las investigaciones disciplinares.

Estos autores diferencian entre la calidad editorial, la calidad científica y el impacto (visibilidad e internacionalización). El choque entre una concepción u otra se produce en este último punto, ya que algunas agencias establecen una correlación directa entre la calidad científica y el impacto obtenido por una revista, es decir, enfatizan la visibilidad como elemento esencial de su calidad. Esta distinción resulta muy interesante porque sabemos que todas las revistas reconocidas cumplen con los requisitos de calidad editorial (formales) y también con los de calidad científica (avalada por ética la revisión por pares de los trabajos publicados), pero muchas de las españolas, aun así, no consiguen un mínimo impacto, pues entran en juego todos los factores a los que nos estamos refiriendo. 
Incluso, cada vez más se empiezan a cuestionar aspectos como la revisión por pares, ya que el notable aumento de trabajos recibidos por las revistas, debido a la creciente presión por publicar, unido al aumento del número de revistas indexadas, implica recurrir a un número mayor de revisores para cumplir con el requisito del doble ciego, lo que dificulta conseguir a los mejores referees. Es paradójico que las revisiones sean desinteresadas y gratuitas y que no estén reconocidas como trabajo académico, mientras que, por el contrario, se acrecientan las situaciones de pagar por publicar o por hacerlo en abierto -como indicábamos anteriormente-, debido al nuevo modelo de negocio hacia el que se decantan algunas editoriales.

A la vez, las agencias nacionales de evaluación, por una lógica política de eficiencia de recursos, están dando más importancia al continente (el impacto de la revista) que al contenido (los artículos). Se supone así que el prestigio del continente avala la calidad de su contenido bajo la premisa de que en las revistas con mayor FI es más difícil publicar, al ser la revisión por pares necesariamente más selectiva. Esto ha generado cierta controversia, pues lo que prevalece en los procesos de evaluación individual del personal docente-investigador son los datos métricos de la revista como medida indirecta de la calidad de su contenido, frente a la valoración de la calidad del propio artículo (Post, 2014). Sin embargo, no se han planteado otras alternativas viables. La tendencia seguida por algunas de estas agencias nacionales se centra prioritariamente en el FI, en particular del JCR de Thomson Reuters y del SJR de Scopus, como elemento determinante y diferenciador de la calidad científica. Se trata, explícitamente, de publicaciones necesarias para obtener una evaluación positiva de la producción investigadora de un profesor o investigador. Estas agencias contemplan otras clasificaciones o rankings (DICE-CINDOC, ERIH, CIRC, FECYT, etc.), aunque en un segundo nivel de importancia, o incluso como aportaciones complementarias, y no suficientes para obtener una evaluación positiva. Quizás a las revistas nacionales presentes en estos dos índices, las agencias las deberían clasificar como de excelencia científica, pero también habría que reconocer la calidad de todas las buenas publicaciones que forman parte de esa clase media de revistas que, sin haber podido acceder a WoS ni a Scopus, están bien gestionadas y presentan también un FI medible en otras métricas, como es Google, MIAR, o como lo fue IN-RECS (Rodríguez-Yunta \& Tejada Artigas, 2013; Giménez-Toledo, 2015).

Si nos atenemos al sistema de evaluación nacional de revistas que ha logrado sobrevivir a la crisis económica (nos referimos al Sello de Calidad de la FECYT), muchas revistas tampoco consiguen el Sello por no pasar el corte determinado por la media de citas en relación con el área. Por tanto, tras la desaparición de IN-RECS, RESH y DICE, o el no reconocimiento de otros sistemas de evaluación existentes, como es el caso de MIAR, o el escaso valor que se da todavía al CIRC, ya no existen las categorías intermedias de revistas: o están en WoS, Scopus (o, con un valor poco definido para la ANECA, en FECYT), o no existen en términos de reconocimiento. Como destaca Alberts (2013), se está produciendo una notable distorsión del IF, que provoca lo que Casadevall y Fang (2014) denominan ahora como impact factor manía (IFmanía). Anteriormente, otros autores se refirieron a este mismo fenómeno como impactolatría (Camí, 1997), o impactitis (Van Diest, Holzel, Burnett \& Crocker, 2001). Es decir, el mal uso del factor de impacto puede sesgar las revistas de determinados campos, ya que 
"existen diferencias sustanciales en la tasa general de cita entre diferentes disciplinas. Las disciplinas con más capacidad de cita (incluyendo la auto-cita) son las ciencias, después las ciencias sociales y por último las humanidades"' (Universo Abierto, 2015, p. 9), en una proporción de 8 a 3. En consecuencia, si se quiere apoyar a esa clase media de revistas y la Administración mantiene una definición de calidad basada en el IF de las revistas, o bien la ANECA reconoce explícitamente el Sello de FECYT y esta establece una delimitación de tramos más flexibles para acceder a la mención de calidad, o bien se crea de nuevo un sistema similar a un SSCI o SJR español, como, por ejemplo, el extinto IN-RECS.

Nunca antes la bibliometría y la cienciometría habían tenido un peso tan grande, ni una transversalidad tan profunda en el ámbito científico. Éste es el criterio diferenciador que distingue a una élite de revistas científicas del resto, a pesar de que una amplia mayoría (las clases medias) adquieran altas cotas de calidad editorial, científica e incluso de visibilidad. El IF no sería tan determinante si no fuera por el extraordinario valor que las agencias de evaluación están dando a que los investigadores publiquen en estas revistas exclusivas y no en otras que también detentan una calidad contrastada, pero sin presencia en los rankings reconocidos (Casadevall \& Fang, 2014). Ello ha generado el conocido dogma publish or perish, que identifica investigar sólo con publicar,

ya que puede suponer logro de notoriedad, prestigio, promoción y reconocimiento personal, aumento de los ingresos, obtención de recursos, etc. Así, damos razón al axioma, publicar para seguir existiendo y prosperar. Además, las instituciones presionan para publicar ... . Por eso han proliferado revistas científicas, algunas de las cuales se lucran con las necesidades de publicar por parte de los docentes universitarios (García Aretio, 2015, p. 9).

En esencia, el problema no es la bibliometría, sino el mal uso que se puede hacer -o se está haciendo- de ella, la falta de comprensión de agencias de evaluación, usuarios y editores de unos indicadores y guarismos tan complejos, e incluso la psicosis que desde algunas instancias se está generando -incluida la propia universidad- para publicar sólo en revistas con IF.

\section{Conclusiones}

Por suerte, existe vida más allá del JCR, ya que "no son pocas las revistas educativas nacionales que atienden a diversos criterios de calidad y que dan difusión a trabajos significativos para la investigación en determinadas áreas para las que algunas revistas, pese a no estar en el JCR, son auténticos referentes" (Fuentes, Luque \& López, 2012, p. 214).

Si no cambian las políticas de evaluación de la producción científica basadas en el IF, se llegará a un cierto darwinismo editorial, en el sentido de que numerosos proyectos se verán obligados a optar por el cierre, la reinvención, la coalición, o continuar con una edición menor o local. El peligro inmediato es que las revistas que hemos clasificado como de clase media, que sobreviven con gran dificultad, desaparezcan si no consiguen entrar en uno de los dos rankings del IF (sólo 6 revistas españolas de educación están 
en JCR y 23 en SJR como hemos indicado anteriormente) o si no encuentran un nicho atractivo por otras vías. La llegada a finales del 2015 de ESCI, nuevo producto de WoS, ha hecho patente una mayor permeabilidad de Thomson Reuters a revistas españolas (catálogo en el que aparecen 29 revistas españolas de educación y que podrían llegar a 60 a finales de 2016 (Galán, 2016), pero la cuestión crucial es si nuestras agencias de evaluación reaccionarán con rapidez y reconocerán a las revistas presentes en este nuevo índice como revistas de calidad.

La falta de competitividad del mundo iberoamericano bajo los criterios de calidad basados en el FI podría cambiar si se aceptan otras métricas o variables para medir este impacto. Hasta el momento, la prevalencia de diversos criterios de calidad asimétricos, entre los que destaca el inglés como principal lengua de comunicación científica, está complicando mucho más la situación para la gran mayoría de revistas de ciencias sociales. Valga como referente que la presencia en el SSCI de revistas no anglosajonas que conservan su lengua nacional es de solo un 7,1 \%, lo que muestra una clara desigualdad de oportunidades para competir, si analizamos los criterios para el cálculo del FI (Ruíz-Corbella et al., 2015).

Parece necesario, en consecuencia, que los países iberoamericanos defiendan sus propios sistemas de evaluación de revistas científicas, como CIRC, el Sello de Calidad de FECYT, SCIELO o REDALyC, creando un FI iberoamericano complementario a JCR y Scopus, que sea reconocido por sus universidades y sus propias agencias de evaluación.

Países como España, México, Brasil, Colombia y Chile tienen políticas de incentivos que premian la publicación en determinadas revistas, pero en algunos de ellos como España y México, los incentivos pensados inicialmente como complementos salariales se han convertido con el tiempo en requisitos necesarios para la promoción profesional, generando confusión y frustración (Acosta, 2014). De la flexibilidad de dichas políticas y de su capacidad para considerar métricas alternativas al IF (como almetrics u otras métricas nacionales o iberoamericanas de impacto tipo IN-RECS) en campos con una fuerte presencia y vinculación social como es la educación, depende, en buena medida, de que las revistas de clase media sobrevivan.

En consecuencia, hay que afrontar cómo difundir y afianzar la investigación a partir de una comunicación compartida, en la que las revistas logren ser verdaderos canales de desarrollo científico que incidan y lleguen a todos los sectores. La visibilidad que favorecen el Social Media potencia también la participación de las redes científicas con el objeto de consolidar reputación, prestigio, citas, etc. Un ejemplo en el área de las ciencias es arXiv, plataforma en el que los propios expertos intercambian artículos y no se llega a publicar hasta que no ha sido valorado por pares en este canal (Rey, 2015). Resulta lógico que cada área de conocimiento tenga su propia cultura, pero no hay duda de que en las ciencias sociales y, en concreto, en educación, debemos dar un salto cualitativo en el modo de trabajo.

\section{Referencias}

Abadal, E., \& Rius, Ll. (2006). Revistas científicas digitales: características e indicadores. Revista de Universidad y Sociedad del Conocimiento, 3(1), 1-20. 
Acosta, A. (2014). Gobierno universitario y comportamiento institucional: la experiencia mexicana. Bordón. Revista de Pedagogía, 66(1), 31-44. doi: 10.13042/Bordon.2014.66102

Alberts, B. (2013). Impact factor distortions. Science, 340(6134), 787, doi: 10.1126/ science. 1240319

Aliaga, F. M. (2014). Veinte años de publicación electrónica y de acceso abierto: la madurez de una pionera. RELIEVE, 20(1), doi: 10.7203/relieve.20.1.3856

Camí, J. (1997). Impactolatría, diagnóstico y tratamiento. Medicina clínica, 109, 515-524. Recuperado de http://goo.gl/W5GO6o_

Casadevall, A., \& Fang, F.C. (2014). Causes for the persistence of impact factor mania. mBio, 5(2). doi: 10.1128/mBio.00064-14

Chavarro, D. (2013). ¿Son los sistemas de indexación y resumen un indicador de la buena calidad editorial de las revistas académicas? Actas del IX Congreso Iberoamericano de Indicadores de Ciencia y Tecnología. Bogotá, Colombia: Observatorio Iberoamericano de la Ciencia, la Tecnología y la Sociedad. Recuperado de http://goo.gl/XoVGa8

Delgado, E., Ruiz-Pérez, R., \& Jiménez-Contreras, E. (2006). La Edición de Revistas Científicas Directrices, Criterios y Modelos de Evaluación. Granada: EC3-Universidad de Granada.

D’Antonio, S. (2014). Algunas reflexiones en torno al acceso abierto, revistas científicas y el campo de publicación académica. Argumentos de razón técnica: Revista española de ciencia, tecnología y sociedad, y filosofía de la tecnología, 17, 173-187

European Commission (2014). Science 2.0': Science in transition. Bruselas, Bélgica: Unión Europea. Recuperado de https://goo.gl/3vC4jO

European Commission (2015). Validation of the results of the public consultation on Science 2.0: Science in Transition. Bruselas, Bélgica: Unión Europea. Recuperado de https:// goo.gl/LSXlua

Galán, A. (2014). Evaluación de la producción científica en educación. ¿Una nueva crisis?. Bordón. Revista de Pedagogía, 66(2), 7-10.

Galán, A. (2016). Volviendo al ESCI (EMERGING SOURCES CITATION INDEX) desde el pensamiento de WoS. Aula Magna 2.0. Recuperado de http://cuedespyd. hypotheses.org/1250

García Aretio, L. (2015). Publicar (casi exclusivamente) en revistas de impacto. Revista Iberoamericana de Educación a Distancia. 18(2), 7-22. doi: http://dx.doi.org/10.5944/ ried.18.2.14254

Giménez-Toledo, E. (2014). Imposturas en el ecosistema de la publicación científica. Revista de Investigación Educativa, 32(1), 13-23. doi: http://dx.doi.org/10.6018/ rie.32.1.190251

Giménez-Toledo, E. (2015). La evaluación de la producción científica: breve análisis crítico. RELIEVE, 21(1), 1-9. doi: http://dx.doi.org/10.7203/relieve.21.1.5160

Giménez-Toledo, E., \& Tejada-Artigas, C.M. (2014). Proceso de publicación, calidad y prestigio de las editoriales científicas en Educación. Educación XX1, 18(1), 17-44. doi: http://dx.doi.org/10.594/educXX1.18.1.12310

Hernández Pina, F., \& Maquilón, J.J. (2010). Indicadores de calidad de las revistas científicas y sistema de gestión editorial mediante OJS. Revista de Investigación Educativa, 28(1), 13-29. 
Hicks, D., Wouters, P., Waltman, L., De Rijcke, S., \& Rafols, I. (2015). The Leiden Manifesto for research metrics. Nature, 520(7548), 429-431, doi: 10.1038/520429a

Finch Working Group (2012). Report of the Working Group on Expanding Access to Published Research Findings. Recuperado de http://goo.gl/819mCw

Fuentes, J.L., Luque, D., \& López, E., (2013). Análisis bibliométrico de las revistas españolas de educación incluidas en el Journal Citation Report. Producción científica y elementos controvertidos. Teoría de la Educación. Revista Interuniversitaria, 24(1), 183-217.

Ley 14/2011, de 1 de junio, de la Ciencia, la Tecnología y la Innovación, BOE 2 junio de 2011. Recuperado de https://goo.gl/dO2bWv

López-Torres, J. (2015). Pagar por publicar en revistas científicas. Revista Clínica de Medicina de Familia. 8(3), 179-181. doi: 10.4321/S1699-695X2015000300001

Mañana-Rodríguez, J., \& Giménez-Toledo, E. (2015). Revistas de educación e investigación educativa. Modelos de negocio y desempeño bibliométrico. Revista de educación, 369, 31-58. doi: 10.4438/1988-592X-RE-2015-369-289

Mendoza, S., \& Paravic, T. (2006). Origen, clasificación y desafíos de las revistas científicas. Investigación y Postgrado, 21(1), 49-75.

Nassi-Caló, L. (2013). Quanto custa publicar em acesso aberto?. SciELO em Perspectiva. Recuperado de http://goo.gl/6Jd2MT

Post, D. (2014). The future of education research publishing: Challenges and responses. Education Policy Analysis Archives, 22(26), 1-9. doi: 10.14507/epaa.v22n26.2014_

Rey, A. (2015). Reputación académica y publicaciones de libre acceso. Educación, Innovación Social, Lecturas, Tendencias, Wikiinnovación. Recuperado de http://goo. $\mathrm{gl} / \mathrm{oJSgFt}$

Rodríguez-Yunta, L., \& Giménez-Toledo, E. (2013). Fusión, coedición o reestructuración de revistas científicas en humanidades y ciencias sociales. El profesional de la información, 22(1), 36-45. doi: 10.3145/epi.2013.ene.05

Rodríguez-Yunta, L., \& Tejada Artigas, C. M. (2013). El editor técnico: un perfil necesario para la profesionalización de la edición de revistas científicas en el entorno digital. Anales de Documentación, 16(2), 1-9. doi: 10.6018/analesdoc.16.2.176391

Rozemblum, C., Unzurrunzaga, C., Banzato, G., \& Pucacco, C. (2015). Calidad editorial y calidad científica en los parámetros para inclusión de revistas científicas en bases de datos en Acceso Abierto y comerciales. Palabra Clave (La Plata), 4(2), 64-80. Recuperado de http://goo.gl/Ywic23

Ruíz-Corbella, M., Galán, A., \& Diestro, A. (2014). Las revistas científicas de Educación en España: evolución y perspectivas de futuro. RELIEVE, 20(2), 1-27. doi: 10.7203/ relieve.20.2.4361

Ruíz-Corbella, M., Galán, A., \& Diestro, A. (2015). Las revistas científicas en Educación ante las exigencias de la sociedad en red. History of Education E Children's Literature, 10(1), 65-71.

Ruíz-Pérez, R., Martín-Martín, A., \& Delgado López-Cózar, E. (2015). Las revistas universitarias en el marco de los criterios de evaluación de la actividad investigadora en España. Revista española de Documentación Científica, 38(2), 1-13. doi: 10.3989/ redc.2015.2.1191 
Sánchez Perona, J. (2015). La peligrosa deriva de las publicaciones en acceso abierto. Ciencia con futuro. Recuperado de http://goo.gl/Td8khR

Torres-Salinas, D. (septiembre, 2008). El paradigma 2.0 en las grandes revistas científicas. Trabajo presentado en 3rd International LIS-EPI Meeting, Valencia.

Universo Abierto (2015). Variaciones en los tipos de citas a través de disciplinas, tipos de documentos, trayectorias y perfiles profesionales. Recuperado de http://goo.gl/ TluXC4

Vessuri, H., Guédon, J. C., \& Cetto, A. M. (2013). Excellence or quality? Impact of the current competition regime on science and scientific publishing in Latin America and its implications for development. Current Sociology, 62(5), 647-665. doi: $10.1177 / 0011392113512839$

Van Diest, P. J., Holzel, H., Burnett, D., \& Crocker, J. (2001). Impactitis: new cures for an old disease. Journal of clinical pathology, 54(11), 817-819.

Villarroya, A., Claudio-González, M., Abadal, E., \& Melero, R. (2012). Modelos de negocio de las editoriales de revistas científicas: implicaciones para el acceso abierto. El profesional de la información, 21(2), 129-135. doi: 10.3145/epi.2012.mar.02

Fecha de recepción: 28 de Noviembre de 2015

Fecha de revisión: 28 de Noviembre de 2015

Fecha de aceptación: 20 de Mayo de 2016 